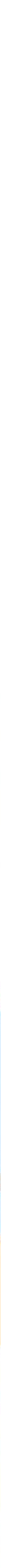


*Pós-graduanda em Enfermagem. Escola de Enfermagem de Ribeirão Preto da Universidade de São Paulo (USP). Ribeirão Preto, São Paulo, Brasil.

E-mail: carolina.grigolato. viola@usp.br

**Professora na Escola de Enfermagem de Ribeirão Preto da USP. Ribeirão Preto, São Paulo, Brasil.

E-mail: rcamargo@eerp.usp.br

*** Pós-graduanda em Enfermagem. Escola de Enfermagem de Ribeirão Preto da USP. Bonfim Paulista, São Paulo, Brasil.

E-mail: isabela.gouvea@usp.br

****Professora na Escola de Enfermagem de Ribeirão Preto da USP. Ribeirão Preto, São

Paulo, Brasil.

E-mail: fersngoes@eerp.usp.br

Recebido para publicação em: 24.9.2016

Aprovado em: 8.8.2017

\section{RELAÇÕES ENTRE A EDUCAÇÃO BÁSICA E O CONHECIMENTO ESPECÍFICO NA EDUCAÇÃO PROFISSIONAL DE NÍVEL TÉCNICO EM ENFERMAGEM}

\section{RELATIONS BETWEEN BASIC EDUCATION AND SPECIFIC KNOWLEDGE IN TECHNICAL-LEVEL VOCATIONAL EDUCATION IN NURSING}

\section{RELACIONES ENTRE LA EDUCACIÓN BÁSICA Y EL CONOCIMIENTO ESPECIIFICO EN LA FORMACIÓN PROFESIONAL DE NIVEL TÉCNICO EN ENFERMERÍA}

Carolina Grigolato Viola*

Rosangela Andrade Aukar de Camargo** Isabela Barbuzano Gouvêa*** Fernanda dos Santos Nogueira de Góes****

\section{Resumo}

Este artigo identificou a trajetória e o desempenho de alunos da educação técnica de nível médio em Enfermagem, a partir da perspectiva dos estudantes de três instituições de ensino de Ribeirão Preto, SP. Não foi comprovada a hipótese de que dificuldades advindas da educação básica interfiram na aprendizagem de enfermagem. A partir daí, faz-se uma reflexão sobre o contexto atual da educação profissional de nível médio em Enfermagem no Brasil e as inter-relações com a sociedade, apreendendo a relação entre o curso de Enfermagem e a qualidade da formação do técnico em enfermagem no Brasil.

Palavras-chave: Enfermagem. Educação profissional. Ensino fundamental.

\section{Abstract}

This article identified the path and performance of students from mid-level technical education in Nursing, from the student perspective in three teaching institutions in Riberão Preto, SP. The hypothesis that difficulties resulting from basic education would interfere with learning in nursing was not proven. Based on this, 
the text reflects on the current context of mid-level vocational education in Nursing in Brazil and its interrelations with society, including the relation between the Nursing program and the quality of technical education in nursing in Brazil.

Keywords: Nursing. Vocational education. Basic education.

\section{Resumen}

Este artículo identificó la trayectoria y el desempeño de alumnos de la educación técnica de nivel medio en Enfermería, a partir de la perspectiva de los estudiantes de tres instituciones de enseñanza de Ribeirão Preto, SP. No se comprobó la hipótesis de que dificultades provenientes de la educación básica interfieren en el aprendizaje de enfermería. A partir de allí, se realiza una reflexión sobre el contexto actual de la formación profesional de nivel medio en Enfermería en Brasil y las interrelaciones con la sociedad, aprehendiendo la relación entre el curso de Enfermería y la calidad de la formación del técnico en enfermería en Brasil.

Palabras clave: Enfermería. Formación profesional. Educación básica.

\section{Introdução}

Dados do Instituto Brasileiro de Geografia e Estatísticas (IBGE), em 2010, demonstraram que aproximadamente $80 \%$ dos profissionais de enfermagem que atuam no País são de nível técnico, denotando a representatividade dessa força de trabalho na Saúde. No estado de São Paulo, no mesmo ano, de um total de 376.135 profissionais licenciados, 55\% eram auxiliares de enfermagem, 24,75\% eram técnicos em enfermagem, e somente 19,43\%, enfermeiros (LUZ, 2010).

É significante a ressalva de que o ensino médio, em que a educação profissional técnica em Enfermagem se enquadra, tem sido historicamente um dos níveis de mais difícil enfrentamento no que diz respeito a sua concepção, estrutura e organização, por conta da sua natureza de mediação entre o ensino fundamental e a formação profissional (GÖTTENS; ALVES; SENA, 2007). Traz uma proposta pedagógica ambígua, que nem sempre atende às finalidades de aprofundamento dos conhecimentos adquiridos no ensino fundamental juntamente com a preparação para o trabalho.

Nesse sentido, estudo realizado com alunos e professores de uma instituição de ensino que oferece curso técnico em Enfermagem demonstrou que 
as principais necessidades de aprendizagem dos alunos estão centradas em conteúdos relacionados a cálculo, gramática, ortografia e interpretação de texto, e não propriamente a conteúdos específicos de enfermagem (GÓES et al., 2015).

As observações feitas podem ter impacto direto e significativo no ensino técnico de Enfermagem, pois o conhecimento construído ao longo da educação básica é necessário para a aprendizagem de conteúdos específicos de enfermagem.

O Pisa - Programa Internacional de Avaliação de Estudantes, avaliação feita pela Organização para a Cooperação e o Desenvolvimento Econômico (OCDE), mediu o desempenho dos estudantes em leitura, matemática e ciências em 65 países. Apesar de o Brasil ter avançado no ranking desde os anos 2000, o país ficou na 54a posição em 2012 (ORGANISATION FOR ECONOMIC CO-OPERATION AND DEVELOPMENT, 2014).

Cabe à educação profissional reforçar e aplicar os conhecimentos advindos da educação básica
O Pisa mostra que há desafios em relação ao aprendizado de matemática. Na área, são seis os níveis de proficiência, e pouco mais de $60 \%$ dos estudantes brasileiros estão no nível 1 ou abaixo dele. Em leitura, o Brasil subiu no ranking, colocando o País no mesmo patamar da Colômbia, da Tunísia e do Uruguai, contudo, abaixo da média da OCDE (ORGANISATION FOR ECONOMIC CO-OPERATION AND DEVELOPMENT, 2014).

Na perspectiva do ensino médio, as escolas profissionalizantes têm um grande desafio a enfrentar, pois caberá à escola técnica complementar a formação anterior, se necessário. À educação profissional cabe mais do que conferir um diploma, pois trata-se de uma etapa educacional que articula o ensino básico à formação para a vida profissional, a partir de uma visão ampla e contextualizada da educação. Cabe à educação profissional reforçar e aplicar os conhecimentos advindos da educação básica que tragam subsídios para o domínio de novos conhecimentos que se fazem necessários à formação profissional de qualidade.

Diante do exposto, questiona-se: como os conhecimentos da educação básica colaboraram para a compreensão de conhecimentos específicos do campo da Enfermagem/Saúde na formação de técnicos e auxiliares de enfermagem? Pela importância dos auxiliares e técnicos em enfermagem para o sistema de saúde brasileiro, a complexidade de sua formação e da relação estreita entre a educação básica e o ensino profissional, este estudo se propõe a identificar a trajetória de formação na educação básica, especificamente nas disciplinas de português e matemática, de alunos da educação profissional técnica em Enfermagem e seu desempenho na educação profissional. 
Este estudo tem como hipótese que há relação entre a qualidade da formação na educação básica e a formação no ensino profissional de nível médio em Enfermagem.

\section{Material e métodos}

O estudo foi aprovado pelo Comitê de Ética em Pesquisa envolvendo seres humanos, CAAE 35015214.6.0000.5393, e seguiu a legislação que regulamenta a pesquisa envolvendo seres humanos (CONSELHO NACIONAL DE SAÚDE, 2013).

Foi realizado estudo exploratório (PEREIRA, 2002), para o qual foram convidadas quatro instituições de educação profissional técnica em Enfermagem, e três aceitaram participar.

Após o aceite das instituições, foi solicitada autorização para contatar os estudantes da educação profissional de nível médio em Enfermagem. No momento da coleta de dados, a escola de educação privada "1" (EP1) contava com oito turmas, somando 92 alunos, nos períodos da manhã e da noite; a escola privada "2" (EP2) oferecia apenas uma turma com 24 alunos; a escola pública "3" (EP3) dispunha de uma turma com 37 alunos. Para ser convidado a participar do estudo, o aluno deveria estar regularmente matriculado no curso e ter cumprido, pelo menos, $50 \%$ do total de atividades.

Para atingir o objetivo proposto, os estudantes preencheram um questionário sobre idade, gênero, local de estudo (ensino público, privado, misto), trabalho (durante a educação básica e atual, na área da Saúde e/ou Enfermagem), tempo de conclusão do ensino fundamental e/ou médio; reprovação no ensino fundamental e/ou médio, dedicação aos estudos, percepção dos alunos sobre alguma dificuldade mesmo após a conclusão dos estudos. Cabe ressaltar que o questionário foi testado previamente por meio de coleta, com dois participantes que não foram incluídos na amostra final.

Após a primeira etapa de coleta, foi solicitado à instituição de ensino o acesso às notas do histórico escolar da educação básica e também às notas nas disciplinas do curso técnico de Enfermagem cursadas até o momento, as quais foram anotadas em formulário específico.

A coleta de dados foi realizada entre os meses de agosto de 2013 e novembro do ano de 2014; justifica-se o longo período de tempo pela dificuldade de contatar a secretaria de uma das instituições participantes. 


\section{Análise dos dados}

Os dados foram digitados duplamente no programa SPSS para Windows. Para avaliar a hipótese de normalidade das variáveis das notas das disciplinas de Português e Matemática bem como as Notas do Ensino Técnico e da variável Idade foi utilizado o teste de Shapiro-Wilk. A não rejeição da hipótese implica no uso de testes paramétricos, enquanto que a rejeição implica em testes não paramétricos.

Para avaliar a existência de correlação entre as notas das disciplinas de Português e Matemática com as Notas do Ensino Técnico e a Idade foi utilizado o coeficiente de correlação de Pearson (paramétrico) ou Spearman (não paramétrico).

Para testar a diferença nas médias das notas de Português, Matemática e Técnico pelas variáveis no Ensino Fundamental e no Ensino Médio, foi utilizado o teste t de Student, no caso paramétrico, ou Mann-Whitney, no caso não paramétrico.

No caso da diferença nas médias das notas de Português, Matemática e do Ensino Técnico pelo Local de Estudo e Tipo de Escola, foi utilizada a Análise de Variância (Anova), no caso paramétrico, ou o teste de Kruskal-Wallis, no caso não paramétrico. Em caso de rejeição da hipótese nula de igualdade, foi utilizado o procedimento de Comparações Múltiplas de Tukey, no caso paramétrico, e de Dunn, no caso não paramétrico.

O nível de significância adotado em todas as análises foi de 5\% (alfa = 0,05). As análises foram realizadas com os programas SPSS versão 22 e $\mathrm{R}$ versão 3.1.2.

\section{Resultados}

Entre as três escolas que aceitaram participar da pesquisa, duas eram privadas e uma pública. No momento da coleta de dados na escola EP1 privada, existiam duas turmas do curso Técnico em Enfermagem, que foi reativado há três anos. Já a escola EP2 é a maior escola de curso Técnico em Enfermagem da cidade e oferecia apenas curso nessa modalidade; e a EP3 era a única escola pública e contava apenas com uma turma.

De um total de 136 alunos elegíveis, 73 participaram do estudo, sendo 15 da escola EP1, 33 da EP2 e 25 da EP3 (Quadro 1). 


\section{Quadro 1 - Caracterização dos estudantes participantes do estudo}

\begin{tabular}{|c|c|c|}
\hline \multicolumn{2}{|c|}{ Dados de caracterização } & n. \\
\hline \multirow{6}{*}{ Idade (anos) } & $16-20$ & 13 \\
\hline & 21 a 24 & 15 \\
\hline & 25 a 29 & 14 \\
\hline & 30 a 34 & 12 \\
\hline & 35 a 39 & 08 \\
\hline & $>40$ & 11 \\
\hline \multirow{2}{*}{ Gênero } & Feminino & 57 \\
\hline & Masculino & 16 \\
\hline \multirow{2}{*}{ Trabalha } & Sim & 49 \\
\hline & Não & 24 \\
\hline \multirow{2}{*}{ Trabalho na área da Saúde } & $\operatorname{Sim}$ & 34 \\
\hline & Não & 39 \\
\hline \multirow{2}{*}{ Trabalho na Enfermagem } & Sim & 9 \\
\hline & Não & 64 \\
\hline \multirow{3}{*}{$\begin{array}{l}\text { Tempo de conclusão do } \\
\text { ensino médio }\end{array}$} & 1 a 5 anos & 34 \\
\hline & 6 a 10 anos & 14 \\
\hline & $>10$ anos & 25 \\
\hline \multirow{2}{*}{ Reprovas no ensino fundamental } & Sim & 16 \\
\hline & Não & 57 \\
\hline \multirow{2}{*}{ Reprovas no ensino médio } & Sim & 12 \\
\hline & Não & 61 \\
\hline
\end{tabular}

Fonte: Elaborado pelos autores.

Nas tabelas a seguir, são apresentadas as médias de Português (Tabela 1) e Matemática (Tabela 2) dos alunos dos cursos de educação profissional de nível médio em Enfermagem.

\section{Tabela 1 - Notas de Português de estudantes da educação} profissional de nível médio em Enfermagem

\begin{tabular}{ccccccccc}
\hline Local & \multicolumn{2}{c}{ Português } & Local & \multicolumn{2}{c}{ Português } & \multicolumn{2}{c}{ Local } & \multicolumn{2}{c}{ Português } \\
\hline & média & 6,76 & & média & 6,45 & & média & 6,86 \\
\multirow{2}{*}{ EP1 } & mediana & 7,00 & \multirow{2}{*}{ EP2 } & mediana & 6,30 & & mediana & 7,00 \\
& mínimo & 6,00 & & mínimo & 5,00 & & mínimo & 5,50 \\
& máximo & 8,00 & & máximo & 9,00 & & máximo & 9,10 \\
\hline
\end{tabular}

Fonte: Elaborada pelos autores. 


\section{Tabela 2 - Notas de Matemática de estudantes da educação profissional de nível médio em Enfermagem}

\begin{tabular}{ccccccccc}
\hline Local & \multicolumn{2}{c}{ Matemática } & Local & \multicolumn{2}{c}{ Matemática } & \multicolumn{2}{c}{ Local } & \multicolumn{2}{c}{ Matemática } \\
\hline & média & 6,53 & & média & 5,81 & & média & 6,53 \\
\multirow{2}{*}{ EP1 } & mediana & 7,00 & & mediana & 5,60 & & mediana & 6,50 \\
& mínimo & 6,00 & EP2 & mínimo & 1,83 & & mínimo & 5,00 \\
& máximo & 7,00 & & máximo & 8,30 & & máximo & 6,53 \\
\hline
\end{tabular}

Fonte: Elaborada pelos autores.

A Tabela 3 apresenta a média de notas dos alunos nas disciplinas específicas da formação em Enfermagem. Cabe destacar que se trata de média simples entre a soma de notas de todas as disciplinas cursadas pelos alunos no curso técnico até o momento da pesquisa em cada uma das escolas.

\section{Tabela 3 - Média de notas específicas de enfermagem dos estudantes da educação profissional de nível médio em Enfermagem}

\begin{tabular}{cccc}
\hline Local do estudo & Número total de alunos & Média & Desvio padrão \\
\hline EP1 & 15 & 6,58 & 0,44674 \\
EP2 & 33 & 8,12 & 0,82445 \\
EP3 & 25 & 7,88 & 0,83166 \\
Total & 73 & 7,72 & 0,96021 \\
\hline
\end{tabular}

Fonte: Elaborada pelos autores.

No que se refere ao local de estudo e média de notas dos alunos, houve diferença estatisticamente significante $(p<0,000)$ entre a escola privada EP1 e as demais escolas participantes do estudo EP2 e EP3.

O tipo de escola que o aluno frequentou não influenciou as notas de Português, Matemática e do curso Técnico (Tabela 4). Contudo, 88,7\% dos alunos frequentaram exclusivamente escola pública.

\section{Tabela 4 - Média de notas dos alunos segundo tipo de escola no ensino fundamental e/ou médio}

\begin{tabular}{lcc}
\multicolumn{1}{c}{ Tipo de escola } & Número total de alunos* & Média \\
\hline Exclusivamente em escola pública & 63 & 7,76 \\
Exclusivamente em escola privada & 5 & 7,80 \\
Escola pública e privada & 3 & 6,78 \\
Total & 71 & 7,73 \\
\hline
\end{tabular}

*Dois alunos não responderam essa questão.

Fonte: Elaborada pelos autores.

B. Téc. Senac, Rio de Janeiro, v. 43, n. 3, p. 126-139, set./dez. 2017. 
Para identificar a média geral da correlação entre as notas de português, matemática, média de notas de curso de Enfermagem e idade, foi utilizada o teste de Spearman, para verificar: coeficiente de correlação de Sperman $(\rho)$; ( $\mathrm{P}$ valor) que está relacionado a significância e o (n) número de participantes.

\section{Tabela 5 - Correlação e significância geral entre notas de português, matemática, média de notas específicas de Enfermagem e idade dos estudantes}

\begin{tabular}{|c|c|c|c|c|c|}
\hline & & \\
\hline & & $\begin{array}{c}\text { Média } \\
\text { Português }\end{array}$ & $\begin{array}{c}\text { Média } \\
\text { Matemática }\end{array}$ & $\begin{array}{c}\text { Média disciplinas } \\
\text { do curso Técnico } \\
\text { Enfermagem }\end{array}$ & $\begin{array}{c}\text { Idade } \\
\text { participantes }\end{array}$ \\
\hline \multirow{3}{*}{$\begin{array}{c}\text { Média } \\
\text { Português }\end{array}$} & $\rho$ & 1,000 & $0,421^{* *}$ & 0,004 & 0,219 \\
\hline & $\mathrm{P}$ valor & -- & 0,000 & 0,972 & 0,070 \\
\hline & $\mathrm{n}$ & 73 & 73 & 71 & 69 \\
\hline \multirow{3}{*}{$\begin{array}{c}\text { Média } \\
\text { Matemática }\end{array}$} & $\rho$ & $0,421^{* *}$ & 1,000 & $-0,123$ & $0,240^{*}$ \\
\hline & $\mathrm{P}$ valor & 0,000 & -- & 0,305 & 0,047 \\
\hline & $\mathrm{n}$ & 73 & 73 & 71 & 69 \\
\hline \multirow{3}{*}{$\begin{array}{l}\text { Média } \\
\text { disciplinas do } \\
\text { curso Técnico } \\
\text { Enfermagem }\end{array}$} & $\rho$ & 0,004 & $-0,123$ & 1,000 & 0,019 \\
\hline & $\mathrm{P}$ valor & 0,972 & 0,305 & -- & 0,876 \\
\hline & $\mathrm{n}$ & 71 & 71 & 73 & 68 \\
\hline \multirow{3}{*}{$\begin{array}{c}\text { Idade } \\
\text { participantes }\end{array}$} & $\rho$ & 0,219 & $0,240^{*}$ & 0,019 & 1,000 \\
\hline & $\mathrm{P}$ valor & 0,070 & 0,047 & 0,876 & -- \\
\hline & $\mathrm{n}$ & 69 & 69 & 68 & 70 \\
\hline
\end{tabular}

Fonte: Elaborada pelos autores.

Pela Tabela 5, pode-se verificar que as notas de Matemática e Português $(0,42)$ e Matemática e idade $(0,24)$ apresentaram correlação positiva e estatisticamente significante, sendo assim, observa-se que há significância quando o (P valor) é menor que 0,05.

Do total de participantes (73), a maioria (49) era trabalhadores, contudo, não houve correlação entre alunos trabalhadores, as notas de Português e Matemática e a média de notas do curso Técnico. Também não houve correlação entre as notas de Português e Matemática e notas do curso Técnico em Enfermagem.

\section{Discussão}

No Relatório Mundial de Saúde de 2006, a Organização Mundial da Saúde (OMS) define os trabalhadores da saúde como todas as pessoas cujo trabalho é proteger e promover o aumento da saúde em suas comunidades (ORGANIZAÇÃO MUNDIAL DE SAÚDE, 2007). Esses trabalhadores, em toda a sua diversidade, formam a força de trabalho global em saúde (ITANI, et al., 
2015). Quando se pensa no cuidado, é necessário associá-lo à educação, principalmente quando se trata de formação de profissionais.

Estudos têm demonstrado que a maioria das instituições que oferecem o curso Técnico em Enfermagem (78,55\%) é privada e com vagas noturnas (CAMARGO et al., 2015; LIMA, 2015b), situação que corrobora os achados deste estudo. Na cidade onde esta pesquisa foi desenvolvida, das três instituições que aceitaram participar, duas eram privadas e, uma, pública; tal situação denota pouco acesso ao ensino na educação profissional de caráter público, situação que pode dificultar a formação profissional da classe trabalhadora (ITANI, et al., 2015; LIMA, 2015a). A criação de vagas em cursos noturnos tem atendido ao perfil dos alunos que buscam a formação profissional de nível médio, os quais são trabalhadores (LIMA, 2015b; OLIVEIRA et al., 2007).

Outro aspecto observado é a presença do gênero feminino, a qual tem sido dominante em cursos técnicos de Enfermagem (DIAS et al., 2013), sendo esta uma característica sócio-histórica da profissão. A enfermagem brasileira desenvolveu-se como profissão tipicamente feminina, apesar de a enfermagem ser exercida pelos dois sexos já há alguns anos (OLIVEIRA et al., 2007).

Não houve correlação entre as notas de Português e Matemática e notas do curso Técnico em Enfermagem
Este estudo também reafirmou que grande parte dos estudantes eram trabalhadores. Tal situação pode colaborar para a dispersão durante as aulas, em razão do pouco rendimento dos alunos, o que não pode, todavia, ser generalizado (GÓES et al., 2015).

Em contrapartida, os Referenciais Curriculares Nacionais da Educação (BRASIL, 2013) demonstram que a formação curricular deve estimular o aluno a explorar o máximo possível sua autonomia e criatividade, capacidade de gerir, liderar e se comunicar e, acima de tudo, adquirir conhecimento.

Os resultados deste estudo demonstraram que os alunos do ensino profissional em Enfermagem chegam às escolas técnicas com medianas de Matemática entre 6,0 - 7,0 e de Português entre 5,5 - 7,0, ou seja, alunos regulares. Porém, não houve correlação entre as notas de Português e Matemática e notas do curso Técnico em Enfermagem. Não foram encontradas na literatura brasileira estudos semelhantes à presente pesquisa, os quais permitissem comparações e discussões. Assim, os dados serão discutidos com base em estudos gerais sobre avaliação de Português e Matemática realizados no Brasil.

A avaliação do Sistema de Avaliação da Educação Básica realizada em 2015, pelo Instituto Nacional de Estudos e Pesquisas Educacionais Anísio Teixeira (Inep), demonstrou uma queda na pontuação geral nos últimos 20 anos, de 290 pontos para 267, para proficiência em língua portuguesa (INEP, 2016). 
Um estudo buscou comparar três provas de avaliação de Matemática aplicadas no Brasil para alunos do ensino fundamental I e II. Os resultados demonstraram que houve progressos, ao longo dos últimos anos, contudo estes estão concentrados nos níveis mais baixos de proficiência (BENASSI et al., 2015).

Machado (2010) destaca a Matemática e o Português (língua materna) como bases para aquisição dos demais conhecimentos. Essas disciplinas são metalinguagens na aquisição de outros conhecimentos e não podem ser reduzidas uma a outra. Para Benassi et al. (2015), tais conteúdos são pressupostos do sistema educacional para preparar o homem para o mundo, que exige aprender a aprender.

Contudo, os achados deste estudo não permitem comprovar a hipótese de que as dificuldades de formação advindas da educação básica interfiram na aprendizagem de conteúdos introdutórios de enfermagem que, por sua vez, interferem na aquisição de conhecimentos específicos, ou seja, uma cadeia de déficits.

Nesta investigação houve somente correlação entre local de estudo e notas dos alunos no curso técnico; houve diferença estatisticamente significante $(p<0,000)$ entre a escola privada EP1 e as demais escolas participantes do estudo, EP2 e EP3. Provavelmente, a maior influência na formação do aluno é a escola técnica (estrutura, professores, gestão, modelo pedagógico, entre outros fatores), em vez de sua formação pregressa.

O Relatório Mundial de Saúde 2006 - Trabalhando juntos pela saúde (ORGANIZAÇÃO MUNDIAL DA SAÚDE, 2007) apontou alguns aspectos ligados à crise na força de trabalho global de saúde, entre os quais se encontram a formação imprópria ou inadequada dos profissionais de enfermagem, o pouco acesso a informações e conhecimento e insuficientes políticas e práticas para o desenvolvimento dos trabalhadores. Dessa forma, o Estado tem recomendado que a formação e a capacitação de recursos humanos na Enfermagem, especialmente de nível técnico, recebam a atenção das autoridades, especialmente, tendo em vista a qualidade dos serviços do Sistema Único de Saúde (SUS).

Diante disso, há a necessidade de se reformular a educação profissional, para que esses profissionais atuem com qualidade, competência e de acordo com as políticas de saúde vigentes no País. Para isso, o conhecimento teórico e prático contemporâneo é indispensável. Contudo, a enfermagem ainda enfrenta uma grande luta dentro de seu próprio espaço de atuação, principalmente no que se refere à competência profissional, em razão dos cursos aligeirados com foco exclusivo no mercado de trabalho (OTTONELLI; VIERO; ROCHA, 2015).

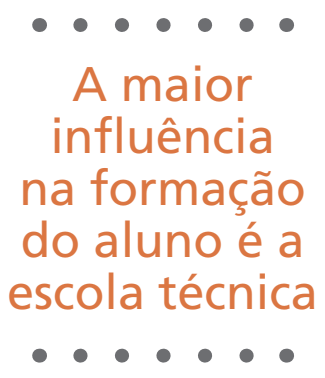

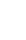


A partir da possibilidade de que as notas dos alunos do curso Técnico dependam de questões relacionadas ao ambiente escolar, os enfermeiros da atenção hospitalar indicaram que os egressos do curso Técnico em Enfermagem não atendem às expectativas do cuidado nos hospitais, pois parte dos técnicos em enfermagem que são contratados nos hospitais tem conhecimento cognitivo, procedimental e atitudinal precário, limitando-se a solucionar atividades mais simples. Também colocam em pauta a qualificação da formação docente e do supervisor de estágios e a falta de melhor planejamento do curso (CAMARGO et al., 2015).

Ainda, para os enfermeiros pesquisados, há a necessidade de se discutir questões pedagógicas, como a seleção dos conteúdos de ensino, as estratégias de ensino e os recursos que cercam cada atividade requerida para cuidar em saúde (CAMARGO et al., 2015).

Diante disso, corrobora-se com Camargo et al. (2015) ao afirmar que a formação de técnicos em enfermagem atende aos parâmetros nacionais de ensino de enfermagem e de acordo com o sistema de saúde vigente no Brasil depende de professores com formação inicial e continuada, que compreendam a importância da articulação entre a teoria e a prática nos diversos ambientes de aprendizagem.

No que se refere à infraestrutura escolar, outro estudo identificou a necessidade de reformas estruturais para tornar o ensino mais atraente, sem as quais se poderia sacrificar a qualidade do perfil do profissional a ser formado, a exemplo de melhoria dos laboratórios de ensino prático e de informática (CAMARGO et al., 2016).

\section{Considerações finais}

desempenho dos alunos do curso Técnico parece ter relação direta com o local de estudo

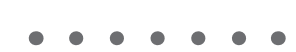

A partir dos achados deste estudo não foi comprovada a hipótese de existirem relações entre a qualidade da formação na educação básica e a formação no ensino profissional de nível médio em Enfermagem.

O desempenho dos alunos do curso Técnico parece ter relação direta com o local de estudo. Assim, pode-se inferir que a qualidade da infraestrutura, os modelos de gestão eficientes para atender às necessidades de saúde brasileiras, o planejamento pedagógico participativo, a utilização de recursos de ensino pautados na formação crítico-reflexiva e a formação inicial e continuada adequada dos professores podem estimular e colaborar para a formação de profissionais técnicos competentes, com capacidades e habilidades atitudinais, procedimentais e cognitivas coerentes com as demandas de suas funções laborais. 
Diante do exposto, faz-se necessária a realização de outros estudos para aprofundamento das questões apontadas nesta pesquisa, com a finalidade de identificar fatores e/ou situações que tenham interferência no processo de formação de profissionais técnicos em Enfermagem.

\section{Referências}

BENASSI, M. T. et. al. Ensino de matemática no ensino fundamental II: as avaliações padronizadas e os resultados brasileiros. Revistas Eletrônicas da Puc-SP, São Paulo, v. 2, n. 1, 2015.

CONSELHO FEDERAL DE ENFERMAGEM (Brasil). Enfermagem em dados. São Paulo, 2013. Disponível em: <http://novo.portalcofen.gov.br/planejamento-estrategico-2>. Acesso em: 21 mar. 2013.

CONSELHO NACIONAL DE SAÚDE (Brasil). Resolução n. 466, de 12 de dezembro de 2012. Diretrizes e normas regulamentadoras de pesquisas envolvendo seres humanos. Diário Oficial da União, Brasília, DF, 13 jun. 2013. Seção 1, p. 59. Disponível em: <http://conselho.saude.gov.br/resolucoes/2012/Res0466.pdf>. Acesso em: 16 abr. 2014.

CAMARGO, R. A. A. et. al. Ambiente de aprendizagem: o espaço escolar da educação profissional técnica de nível médio em enfermagem. Revista Enfermagem UERJ, Rio de Janeiro, v. 24, n. 3, 2016.

CAMARGO, R. A. A. et. al. Avaliação da formação do técnico de enfermagem por enfermeiros da prática hospitalar. Revista Mineira de Enfermagem, Belo Horizonte, v. 19, n. 4, p. 62-68, out./dez. 2015.

DIAS, A. P. et. al. Perfil e perspectivas de alunos na educação profissional técnica de nível médio em enfermagem de uma escola pública do Paraná. FIEP Bulletin, v. 83, 2013. Edição especial.

GÓES, F. S. N. et al. Necessidades de aprendizagem de alunos da educação profissional de nível técnico em enfermagem. Revista Brasileira de Enfermagem, v. 68, n. 1, p. 20-25, jan./fev. 2015.

GÖTTENS, L. D. B.; ALVES, E. D.; SENA, R. R. A enfermagem brasileira e a profissionalização de nível técnico: análise em retrospectiva. Revista Latino-

Americana de Enfermagem, Ribeirão Preto, v. 15, n. 5, p. 1033-1040, 2007.

INEP. Prova Brasil 2015. Brasília, DF, 2016. Disponível em: <http://portal.inep.gov.br/ web/saeb/resultados-2015>. Acesso em: 15 set. 2016.

ITANI, A. et al. Educação e formação profissional: traçando paralelos. Boletim Técnico do Senac: a revista da educação profissional, Rio de Janeiro, v. 41, n. 3, p.10-16, 2015. Disponível em: <http://www.senac.br/conhecimento/boletim-tecnico-do-senac. aspx>. Acesso em: 20 jun. 2016. 
LIMA, Flávia. Os contornos da enfermagem brasileira. RET-SUS: Rede de Escolas Técnicas do SUS, Rio de Janeiro, v. 9, n. 74, p.6-9, out. 2015a. Bimestral. Disponível em: <www.retsus.fiocruz.br>. Acesso em: 20 jul. 2016.

LIMA, Flávia. As desigualdades das relações profissionais. RET-SUS: Rede de Escolas Técnicas do SUS, Rio de Janeiro, v. 9, n. 73, p.4-7, ago. 2015b. Bimestral. Disponível em: <www.retsus.fiocruz.br>. Acesso em: 20 jul. 2016.

LUZ, S. Enfermagem: quantos somos x onde estamos. [S.I.]: Portal da Enfermagem, 2010. Disponível em: <http://www.portaldaenfermagem.com.br>. Acesso em: 15 dez. 2010.

MACHADO, L. V. Avaliação de larga escala e proficiência matemática. Dissertação (Mestrado em Ensino de Matemática) - Instituto de Matemática, Universidade Federal do Rio de Janeiro, 2010. Disponível em: <http://www.pg.im.ufrj. br/pemat/26\%20Ledo\%20Vaccaro.pdf>. Acesso em: 15 ago. 2017.

OLIVEIRA, B. G. R. B. et. al.. Perfil dos alunos ingressos nos cursos de auxiliar e técnico de enfermagem do Projeto de Profissionalização dos Trabalhadores da Área de Enfermagem (PROFAE) no Rio de Janeiro - Brasil. Rev. Latino-Am. Enfermagem. v.15, n.1, pp. 127-133, 2007.

ORGANISATION FOR ECONOMIC CO-OPERATION AND DEVELOPMENT. PISA 2012 results: creative problem solving: students' skills in tackling real-life problems, (volume V). Paris: OECD Publishing, 2014. Disponível em: <http://www.keepeek.com/ Digital-Asset-Management/oecd/education/pisa-2012-results-skills-for-life-volumev_9789264208070-en\#page4>. Acesso em: 15 abr. 2014.

ORGANIZAÇÃO MUNDIAL DA SAÚDE. Relatório mundial de saúde, 2006: trabalhando juntos pela saúde. Brasília, DF: Ministério da Saúde, 2007. Disponível em: $<$ http://www.who.int/eportuguese/publications/pt/>. Acesso em: 4 mar. 2015.

OTTONELLI, J.; VIERO, E. F. F.; ROCHA, K. M. Estudo de caso: metodologia de ensinoaprendizagem na educação profissional. Boletim Técnico do Senac: a revista da educação profissional, Rio de Janeiro, v. 41, n. 3, p. 57-59, 2015. Disponível em: $<$ http://www.senac.br/conhecimento/boletim-tecnico-do-senac.aspx>. Acesso em: 20 jun. 2016.

PEREIRA, M. G. Metodologia: 12 métodos empregados em epidemiologia. In: PEREIRA, M. G. Epidemiologia: teoria e prática. Rio de Janeiro: Guanabara Koogan, 2002. pt. 4, p. 269-288. 